\title{
Spatial and spatial-frequency filtering using one-dimensional graded-index lattices with defects
}

\author{
P.V. Usik ${ }^{a}$, A.E. Serebryannikov ${ }^{\text {b,c,* }}$, Ekmel Ozbay ${ }^{c}$ \\ a Institute of Radio Astronomy, NASU, Acad. Proskury 12, 61085 Kharkiv, Ukraine \\ ${ }^{\mathrm{b}}$ Technische Universitaet Hamburg-Harburg, D-21071 Hamburg, Germany \\ ${ }^{\mathrm{c}}$ Nanotechnology Research Center - NANOTAM, Bilkent University, 06800 Ankara, Turkey
}

\section{A R T I C L E I N F O}

\section{Article history:}

Received 30 January 2009

Received in revised form 10 July 2009

Accepted 24 August 2009

\section{PACS:}

42.70.Qs

42.79.Ry

42.79. Ci

78.20.Ci

Keywords:

Spatial filtering

Graded-index lattice

Defect mode

Fabry-Pérot

\begin{abstract}
A B S T R A C T
The potential of one-dimensional, periodic, graded-index, isotropic dielectric lattices with defects in multiband spatial and spatial-frequency filtering is studied. It is shown that both narrow- and wide-bandpass filters can be obtained at a proper choice of the number, location, and parameters of the defects placed inside the relatively thin slabs. The peculiarities of achieving multibandness for narrow- and wide-bandpass filters are discussed. Multiband narrow-bandpass filtering is closely related to the transmission features that are associated with Fabry-Pérot resonators with semitransparent planar mirrors. Correspondingly, the observed transmission can be interpreted in terms of the equivalent parameters of such resonators. In particular, it is shown that the resonators filled with an ultralow-index medium can be mimicked, so that defect-mode angle-domain spectrum can be rarefied at large angles of incidence. The obtained results are also expected to be applicable for prediction of the angle-domain behavior of transmission in case of piecewise-homogeneous multilayers.
\end{abstract}

(c) 2009 Elsevier B.V. All rights reserved.

\section{Introduction}

Spatial filters have many applications, which are mostly related to image enhancement and information processing and include analysis and modification of spatial spectrum, radar data processing, aerial imaging, biomedical applications, detection of extrasolar planets and so on [1,2]. Spatial-frequency filtering is used for controlling radiation of lasers [3]. Various performances of spatial filters have been suggested, which are based on the use of anisotropic media [1], resonant-grating systems [3,4], interference patterns [5], multilayer stacks combined with a prism [2], and metallic grids [6]. In particular, the possibilities of realizing the low-pass, high-pass, and bandpass filters with wide and steeply bounded pass bands [2] and the narrow-passband filters [3,4] have been demonstrated. Two-dimensional photonic crystals (PC) with [7] and without [8] defects are also known to be appropriate for obtaining various angle-selective transmission effects. In most of the mentioned works, consideration has been restricted

\footnotetext{
* Corresponding author. Address: Technische Universitaet Hamburg-Harburg, D21071 Hamburg, Germany. Tel.: +49 (040) 428783372.

E-mail address: serebryannikov@tu-harburg.de (A.E. Serebryannikov).
}

to single-band spatial filtering. The potential of one-dimensional lattices in multiband spatial filtering is not yet satisfactorily studied.

The goal of this paper is demonstrating the potential of onedimensional periodic lattices with defects in multiband, polarization-sensitive spatial and spatial-frequency filters. The exploited physical mechanism is based on the use of multiple defect modes, which can be either coupled or not coupled, leading to wide- and narrow-bandpass filters, respectively. For the sake of generality, we consider the graded-index slabs rather than piecewise-homogeneous multilayers. It is expected that the principal features observed for the graded-index slabs should remain for the multiulayer stacks with the same index contrast between the alternating layers. The basic difference is that higher $Q$-values are expected to be achievable for individual defect modes in case of multilayers. In turn, the theories of graded-index rugate frequency filters [9-12], thin-film multilayer frequency filters [13], and onedimensional PCs [14-17] should provide one with the initial guidelines for obtaining a desired angle dependence of transmission. However, although the existing frequency-domain transmission results might give some ideas for obtaining a desired spatial filter, they do not allow one understanding some important points like 
angle-domain multibandness. Therefore, consideration at a continuous variation of the incidence angle is required.

Piecewise-homogeneous multilayers have been studied in a large number of the works using the last two theories mentioned. The slabs with a graded refractive index $n(x)$ and alternating sign of $d n / d x$ known as rugate filters have been widely used to realize many optical coating functions in the frequency domain, while offerring some advantages as compared to conventional multilayer stacks, like low internal stress, continuous matching of the refraction index, and suppression of sidelobes, e.g., see [18]. The appearance of the sole transmission peak due to setting $d n / d x=0$ $\left(n=n_{\max }\right)$ in the middle of otherwise periodic slab has been demonstrated in [9]. Later, transmission [18] and dispersion [19] have been studied for similar profiles of $n(x)$ showing $n=n_{\text {min }}$, by using the theory of the localized defect modes. We will show that the multiple angle-domain pass bands with different width can be obtained in relatively simple and unified structures, where defects are obtained by "removing" certain profile periods, i.e., by taking $n=n_{\min }$. The obtained pass band shapes are consistent with those being appropriate for spatial filtering, e.g., compare to [1-5]. The examples of the angle(-frequency) behavior of transmittance will be presented and discussed in terms of band width and multibandness. In case of multiband narrow-bandpass filters, two alternative interpretations in terms of equivalent parameters of Fabry-Pérot resonator will be used, that show, in particular, the possibility of mimicking the filling medium and/or distance between the mirrors, which substantially differ from the actual ones. Calculations will be performed using the local reflection model $[20,21]$.

\section{Theoretical background}

Let us consider the one-dimensional graded-index isotropic profiles, for which the coordinate-dependent relative permittivity is set as the superposition of $M$ shifted Gaussian peaks as follows:

$\varepsilon(x)=\varepsilon_{b}+\sum_{m=1}^{M} \alpha_{m} \exp \left[-\left(x-d_{m}\right)^{2} / \beta_{m}\right]$,

where $\varepsilon_{b}$ is the relative permittivity of the background medium, $a$ is lattice constant, $\beta_{m}$ and $d_{m}=d_{0}+a(m-1)$ are the parameters of the Gaussian exponent which determine width and location of the $m$ th peak, and $d_{0}$ is thickness of a matching layer before and after the Gaussian profile. The total thickness of the slab is $D=2 d_{0}+a(M-1)$. Setting $\beta_{m}=B$ and $\alpha_{m}=A$ for all $m$, one obtains a profile that is periodic, except for the edges. In this paper, $A=10.4, B / D^{2}=2 \times 10^{-4}$, and $d_{0} / D=0.06$. This choice provides, in particular, that the range of variation of $\varepsilon(x)$ within the slab corresponds to the materials available at optical frequencies. It is assumed that permeability $\mu=1$ and $\varepsilon_{b}=1$. The required defects can be obtained by removing one to several peaks of $\varepsilon(x)$, i.e., by setting $\alpha_{m}=0$ for some values of $m=m_{h}$, where $h$ means the number of a peak removed, $h=1,2, \ldots, N$, and $N$ is the total number of removed peaks.

To calculate transmittance, we use one of the versions of the local reflection model [20], which leads to the following Riccati equation with respect to the local reflection coefficient $r$ [21]:

$d r / d x=i 2 k \eta r-\left(1-r^{2}\right) \zeta$

where $\zeta=\gamma d \varepsilon / d x, \eta=\left[\varepsilon(x)-\sin ^{2} \theta\right]^{1 / 2}, \gamma=-1 / 4 \eta^{2}$ for $s$ polarization and $\gamma=\left(\eta^{2}-\sin ^{2} \theta\right) / 4 \eta^{2} \varepsilon$ for $p$ polarization, $k=\omega / c$ is freespace wavenumber, and $\theta$ is angle of incidence. Eq. (2) is solved with the boundary condition $r(D)=0$, so that $x$ is varied toward smaller values. Once reflectance $\mathscr{R}=r^{2}(0)$ is found, transmittance $\mathscr{T}$ is calculated by taking into account the energy conservation, i.e., $\mathscr{T}=1-\mathscr{R}$. The integral equation technique from [22] has been used in order to validate the obtained transmission results.
The results existing for two- and three-dimensional PCs with defects, e.g., see $[23,24]$, and for multilayer piecewise-homogeneous stacks [13] were used to properly place defects and estimate a required range of $\varepsilon$ variation within the slab. This is possible since the dominant physics that originates from the presence of the defects is expected to be the same or similar. In particular, it has been shown in [23] that a single wide defect inside a two-dimensional PC works like a multimode Fabry-Pérot resonator, so that multiple peaks of $\mathscr{T}$ do appear, allowing one realization of multiband narrow-bandpass frequency filters. The corresponding frequencies can be tuned in a wide range by tilting the slab. This feature can be explained in terms of non-flat dispersion of the defect modes, i.e., $\left|d \tilde{\omega} / d k_{d}\right| \neq 0$ where $\tilde{\omega}$ is eigen frequency and $k_{d}$ is wavenumber component for the direction being parallel to the defect axis (compare to [23]). It is also known from the theory of thin-film multilayer filters and can be interpreted as the effect of the effective cavity length that is increasing with $\theta$.

According to [24], multiple peaks of transmittance can be obtained at $\theta=0$ in the PCs with multiple small defects. Their appearance can be interpreted in terms of the coupling of individual single-mode resonators. At certain conditions, the neighbouring peaks can merge that results in the forming of a rather wide pass band within a $\omega$-domain band gap [24]. Similar pass bands are known from the theory of thin-film filters based on one-dimensional multilayer piecewise-homogeneous dielectric stacks [13]. Based on these facts, one might expect that in order to obtain a multiband spatial and spatial-frequency filter, either a single defect being several periods $a$ wide, or several narrow defects, e.g., every being one period wide, should be introduced to an otherwise periodic lattice. This is the main difference in the comparison with the single-defect profiles, which were studied in $[9,18]$.

Fig. 1 shows two profiles of $\varepsilon(x)$, which are used in our study. Orientations of the wave vector $(\mathbf{k})$ and electric $(\mathbf{E})$ and magnetic (H) field components correspond here to the $s$ polarized incident wave (note the different meaning of the ordinate for the permittivity and field vector diagram). The first profile is topologically similar to the graded-index profiles considered in $[9,18]$. However, it is distinguished from the profile in [18] in that the more peaks are removed from the middle of the slab, and from the profile in [9] in that $\varepsilon$ tends to $\varepsilon_{\text {min }} \approx \varepsilon_{b}$ rather than to $\varepsilon_{\min } \approx \varepsilon_{b}+A$ within the single wide defect. For the second profile, two non-neighbouring peaks are removed, so that the symmetry plane at $x=D / 2$ remains.
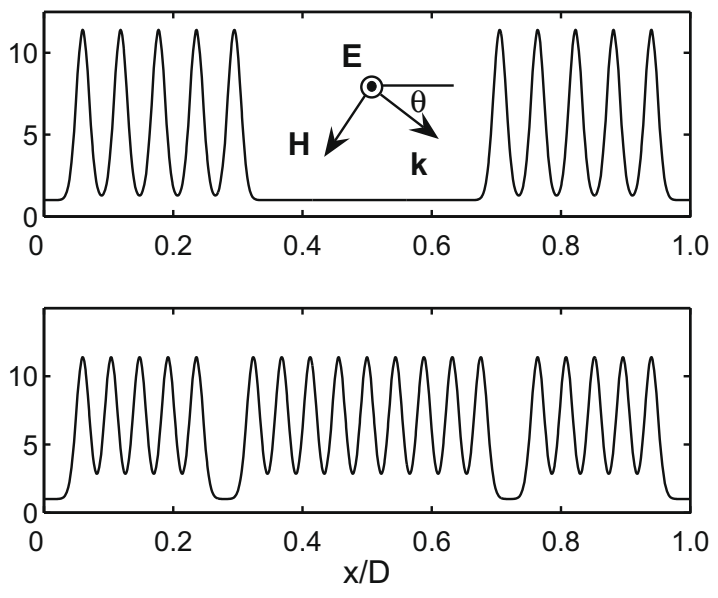

Fig. 1. Relative permittivity $\varepsilon$ as a function of the normalized coordinate $x / D$ : upper plot - $M=16$, six Gaussian peaks from 6 th to 11 th are removed, i.e., $h=6, m_{1}=6, m_{2}=7, \ldots, m_{6}=11$; lower plot $-M=21$, the 6 th and 16 th Gaussian peaks are removed, i.e., $h=2, m_{1}=6$, and $m_{2}=16$; inset - vectors $\mathbf{E}=\mathbf{z} E_{z}$, $\mathbf{H}=\mathbf{x} H_{x}+\mathbf{y} H_{y}$, and $\mathbf{k}$ are shown in $x y z$ coordinate system for $s$ polarized light. 


\section{Results and discussion}

\subsection{Transmission spectra}

We will start from a brief consideration of the typical transmission spectra. In Fig. 2, $\mathscr{T}$ vs $k D$ is presented for the $\varepsilon$-profiles shown in Fig. 1. Here and in the next figures, the large numbers (1 or 2) mean number of a $\omega$-domain stop band (band gap) of the corresponding periodic lattice $\left(\alpha_{m}=A\right.$ for all $m$ ), within which the defect modes do appear. The sharp peaks of $\mathscr{T}=1$ are demonstrated for the first and second stop bands (Fig. 2a), or the first stop band (Fig. 2b), showing different sensitivity to polarization and different location with respect to each other, while $\theta$ is varied. The latter can be realized due to the simultaneous contribution of two or more defect modes.

As a result, location of the peaks in Fig. 2a can almost coincide for $p$ and $s$ polarizations at $\theta>0$, as occurs at $k D=21.67$ and $\theta=40^{\circ}$, or at $\theta=0$ and $\theta=40^{\circ}$ for the same $(s)$ polarization, as occurs at $k D=29.5$. The next remark concerns the $Q$-factor. For the modes occurring at $k D=24.01$ for $\theta=0$, and at $k D=21.67$ and $k D=53.19$ for $\theta=40^{\circ}$ (s polarization), $Q>2 \times 10^{3}$. This is substantially larger than $Q$-values, which were obtained for the rugate profiles studied earlier $[9,18]$. Hence, although the achievable $Q-$ values should be even higher for the multilayer structures with the same $\varepsilon$-contrast, high- $Q$ modes can be obtained using the graded-index technology, too. The $Q$-values of the modes, which correspond to the peaks of $\mathscr{T}$ in Fig. 2a, are comparable with or even higher than those in some performances based on the twodimensional PCs (e.g., see Fig. 5 in [23]).

In Fig. 2b, another expected effect is demonstrated. In the vicinity of $k D=31$, a relatively wide pass band appears due to the coupling of two defect-mode resonators, which is well isolated at $\theta=0$ from the regular pass band arising at $k D>33$. At $\theta=40^{\circ}$, the isolated defect-mode pass band remains for $p$ polarization only. One can see that narrow- and wide-bandpass spatial filters can be realized in the same slab, but within different frequency ranges, i.e., at $k D=23$ and $k D=31$, respectively. It is noteworthy that the symmetry with respect to the midplane $x=D / 2$, i.e., $n(x)=n(D-x)$ at $x \leqslant D / 2$, should guarantee that $\mathscr{T}=1$ at the maxima. Electric field patterns are shown in Fig. 3 in two cases of $\mathscr{T} \approx 1$ for $s$ polarization. Connection of the peaks of $\mathscr{T}$ to the defect modes is clearly seen. Therefore, the boundaries between the layers are not necessarily sharp in order to realize a defect-mode regime. Furthermore, they may be strongly blurred. Despite this, the basic far- and nearfield features, which are usually associated with the piecewisehomogeneous structures, do remain.
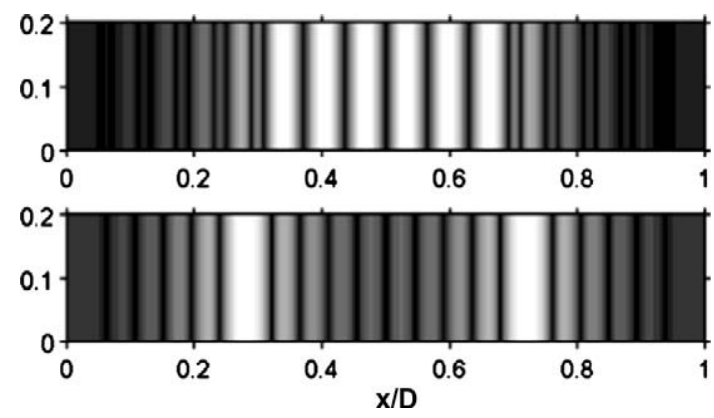

Fig. 3. Electric field at the maxima of $\mathscr{T}$ for the slabs from Fig. 1: upper plot $-\varepsilon(x)$ is taken from the upper plot in Fig. $1, k D=52$ and $\theta=20.63^{\circ}$ (see Fig. 6b), lower plot $\varepsilon(x)$ is taken from the lower plot in Fig. $1, k D=31.58$ and $\theta=20^{\circ}$ (see Fig. 5b); brighter regions correspond to stronger field; ordinate is shown in units of $y / D$.

\subsection{Wide-bandpass filters}

Now consider a typical variation of $\mathscr{T}$ on the $(k D, \theta)$-plane for the $\varepsilon$-profile with the two coupled defect-mode resonators, which is appropriate for obtaining the wide-bandpass spatial and spatialfrequency filters. In line with the goals of this paper, the emphasis is put on the multiband operation rather than on the width and shape of the pass band. Fig. 4 presents an example, where transmission within the defect-mode pass band is shown simultaneously with that for the adjacent part of a regular pass band. Regions shown in white correspond to $t=\mathscr{T}_{s}+\mathscr{T}_{p} \approx 2$, those in mid-gray do to $t \approx 1$. For the sake of convenience, transmission is presented in two plots. The defect-mode pass bands for $s$ and $p$ polarizations can either overlap or do not overlap, depending on subregion on the $(k D, \theta)$-plane.

At $31<k D<31.2$ and small $\theta$, there are subregions where $\mathscr{T}_{s}>0.9$ and $\mathscr{T}_{p}>0.9$ simultaneously. At a bit larger $\theta, \mathscr{T}_{p}>0.9$ while $\mathscr{T}_{s} \approx 0$, so that the $\theta$-domain pass band for $p$ polarization is wider than for $s$ polarization. At $31.2<k D<31.5$, pass bands for two polarizations partially coincide but do not show a common lower boundary at $\theta=0$. The most important regime is realized at $31.7<k D<32.7$, where pass bands for $p$ and $s$ polarizations do not overlap for all $\theta$, i.e., $\mathscr{T}_{s} \approx 0$ or $\mathscr{T}_{p} \approx 0$ beyond the upper and lower pass bands in Fig. 4a, respectively. Finally, at $k D>32.7$, a defect-mode pass band still occurs only for $p$ polarization. The observed difference in the behavior of $\mathscr{T}_{p}$ and $\mathscr{T}_{s}$ can be explained in terms of dispersion. It is qualitatively coincides with the results obtained earlier for multilayer stacks in $\omega$-domain at different $\theta$ values.
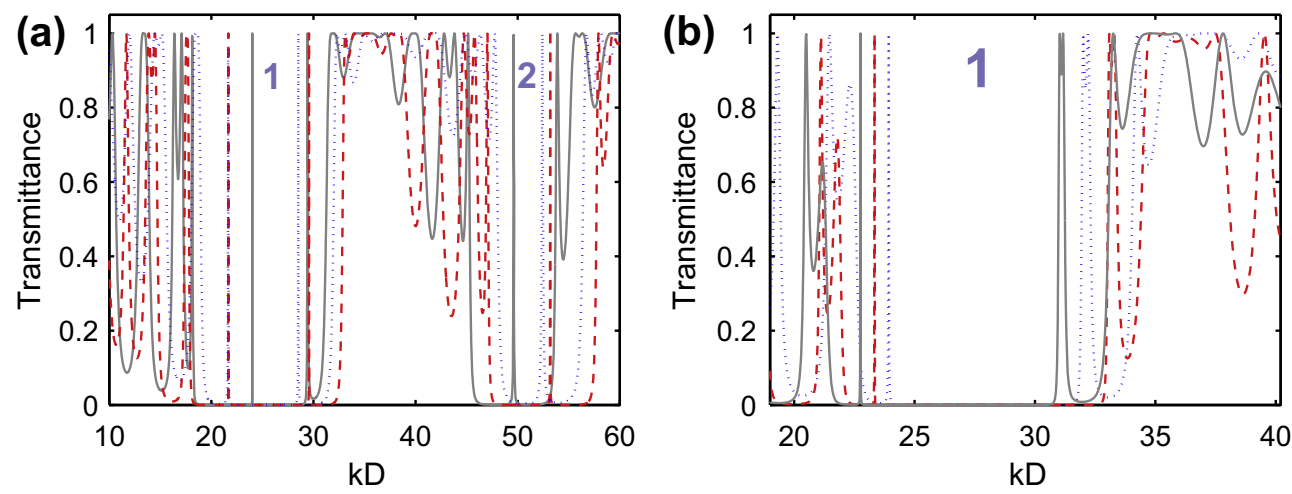

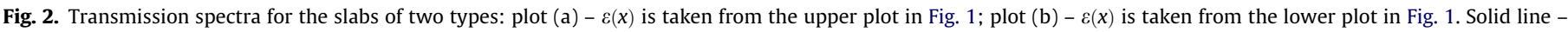
$\theta=0$, dashed line $-\theta=40^{\circ}, s$ polarization; dotted line $-\theta=40^{\circ}, p$ polarization. 

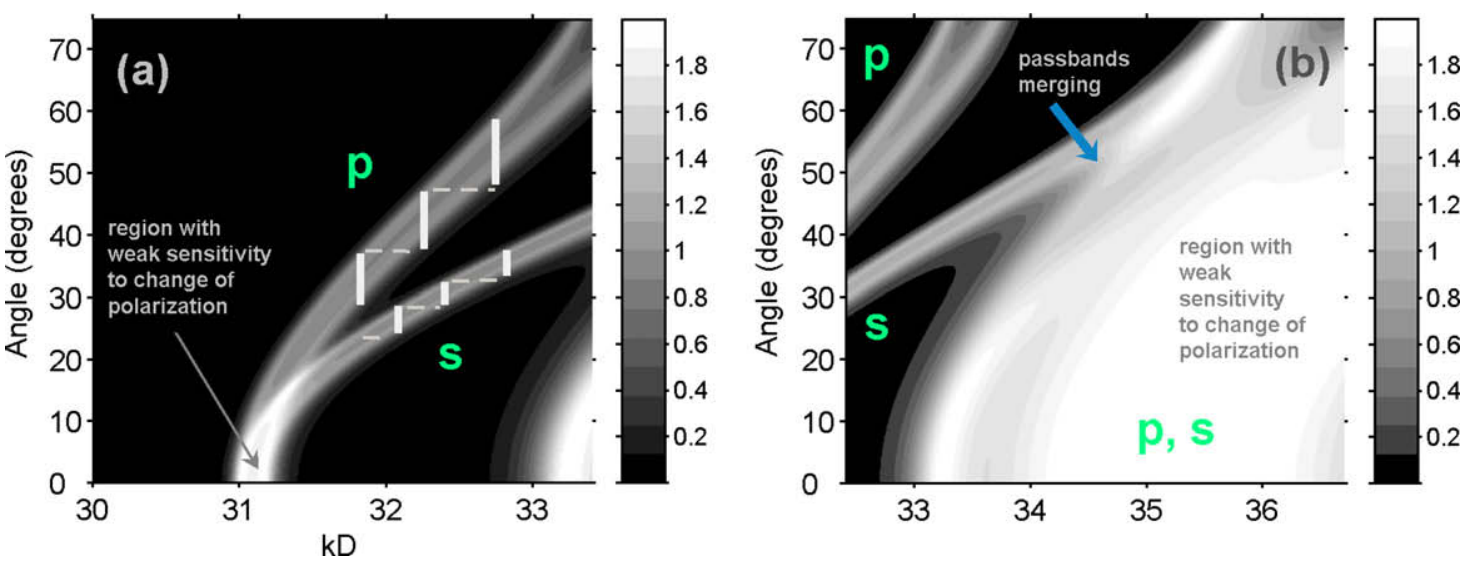

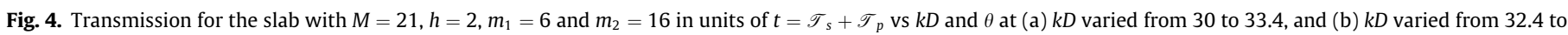
36.7; non-overlapping pass bands are shown in plot (a) by dashed lines.

To obtain an ideal multiband filter, the $\theta$-domain multiple pass bands showing nearly the same band width $W_{\theta}$ within a wide range of variation of $k D$ are required, which satisfy the condition

$d \hat{\omega}_{1} / d \theta \approx d \hat{\omega}_{2} / d \theta \approx C$,

where $\hat{\omega}_{1}$ and $\hat{\omega}_{2}$ mean angular resonance frequencies for the neighboring defect modes, and $C$ is polarization dependent constant. For each polarization, the $\theta$-domain pass bands with a slight overlapping can be obtained simultaneously at several values of $\omega, \omega_{1}<\omega_{2}<\cdots<\omega_{n}$, provided that

$$
\begin{aligned}
\theta_{\max }\left(\omega_{1}\right) & <\theta_{\min }\left(\omega_{2}\right), \theta_{\max }\left(\omega_{2}\right)<\theta_{\min }\left(\omega_{3}\right)<\cdots<\theta_{\max }\left(\omega_{n-1}\right) \\
& <\theta_{\min }\left(\omega_{n}\right)
\end{aligned}
$$

where $\theta_{\min }\left(\omega_{i}\right)$ and $\theta_{\max }\left(\omega_{i}\right)$ mean the lower and upper boundaries of the $\theta$-domain pass band at $\omega=\omega_{i}$, which are chosen by keeping in mind an overlapping that is allowed.

In Fig. 4a, conditions (3) and (4) are approximately satisfied for three $\theta$-domain pass bands for each polarization. They are shown by vertical lines, every corresponding to an unique $\omega$-value. Alternatively, at least three $\omega$-domain pass bands can be obtained (horizontal lines). In the similar manner, multiple $(k D, \theta)$-ranges can be chosen for the purposes of spatial-frequency filtering. In this case, the region of $\mathscr{T} \approx 1$ should be covered, for example, by non-overlapping squares, every corresponding to a certain pass band. The data in Fig. 4b explain why a defect-mode related, isolated pass band appears in Fig. 2b at $\theta=40^{\circ}$ for $p$ polarization only. For $s$ polarization, it corresponds to the region where it merges with a regular pass band. Note that this feature also occurs in various PCs, e.g., in the two-dimensional square-lattice PCs with diameter-to-lattice ratio $d / a=0.4$, relative permittivity of the rods $\varepsilon_{r}=11.4$, line $\operatorname{defect}(\mathrm{s})$, and interfaces being parallel to $\Gamma-X$ direction. Transmission with a slight or vanishing sensitivity to polarization $\left(t>1.8, \mathscr{T}_{s}>0.9\right.$ and $\left.\mathscr{T}_{p}>0.9\right)$ can be obtained, in principle, using either the defect-mode related pass band like that in Fig. 4 a at $k D=31$ or the regular pass band like that in Fig. $4 \mathrm{~b}$ at $33<k D<36$.

Based on the above discussed features, one can distinguish between the following cases, which can be realized in $\theta$-domain: (i) low-pass filtering with different senstivity to polarization within the adjacent ranges of $\theta$ at $\omega=$ const; (ii) two-bandpass filtering at $\omega=$ const, to which the waves of both polarizations are involved; and (iii) multiband bandpass filtering with the pass bands corresponding to different $\omega$, while the waves of the both polarizations are involved to the filtering. These cases are demonstrated in Fig. 5. Using the results presented, one can retrieve the features that are necessary for the obtaining of a large number of $\theta$-domain pass bands. At least two of them should be mentioned. First, the lowest $\hat{\omega}_{j}$ at $\theta=0$ must be located as close as possible to the lower or upper edge of the stop band, provided that $C>0$ or $C<0$, respectively. Second, the smaller $|C|$, and the wider $\omega$-domain stop band, the better is. It is noteworthy that the strong sensitivity of transmission to polarization and, in particular, wide-range suppression of $\mathscr{T}$ for one of polarizations, has been demonstrated in $\omega$-domain for the regular pass bands in PCs without defects (e.g., see Refs. $[25,26])$. However, the mechanism related to the coupled defect modes looks more preferable, at least for obtaining the pass bands, which would be entirely non-overlapping for $p$ and $s$ polarizations.

\subsection{Narrow-bandpass filters}

Now consider the $\theta$-domain transmission for the slabs with a single wide defect, which are expected to be appropriate for obtaining multiband, polarization-sensitive, narrow-bandpass spatial and spatial-frequency filters. Transmission for the filters of this type often show the features, which are considered to be conventional for Fabry-Pérot etalon. In Fig. 6, $\mathscr{T}$ vs $\theta$ is presented for the two values of $k D$, which are taken from the second $\omega$-domain pass band in Fig. 2a. One of the typical features is variation of the peak width $W_{\theta}$ in a wide range. The smallest value of $W_{\theta}$ is here less than $0.05^{\circ}$ for $s$ polarization and about $0.34^{\circ}$ for $p$ polarization. These values are rather close to those obtained in the resonant-grating filters in the reflection mode [4]. For the $s$ polarization, increase of $\theta$ results in decrease of $W_{\theta}$. For the $p$ polarization, a non-monotonous dependence of $W_{\theta}$ on $\theta$ takes place. As a result, the minimal $W_{\theta}$ corresponds to an intermediate $\theta$. The observed tunability of the peak width is similar to that obtained in $\omega$-domain for two-dimensional PCs with a wide defect by varying $\theta$ [23], as well as for other periodic structures with defects.

It is worth noting that the $k D$ value in Fig. 6a corresponds to the maximum of $\mathscr{T}=1$ arising at the edge of the $\omega$-domain regular pass band at $\theta=0$. In this case, behavior of $\mathscr{T}$ vs $\theta$ at $\theta<25^{\circ}$ is typical for the total external reflection, which occurs above a critical $\theta$-value, $\theta \geqslant \theta_{c}$, when the light propagating in vacuum is incident on the interface of a medium with refractive index $0<n<1$ [27]. In Fig. 6a, this behavior co-exists with the multiple defect-mode-inspired peaks, which appear at $\theta_{\max }>\theta>\theta_{\min }$, where $\theta_{\min } \approx \theta_{c}$, and values of $\theta_{\min }$ and $\theta_{\max }$ depend on polarization. In fact, the dependence of $\mathscr{T}$ on $\theta$ for the $s$ polarization is similar to those typical for Fabry-Pérot etalon. For the $p$ polarization, this similarity does not remain at large $\theta$, where $W_{\theta}$ is increased, so that the neighboring peaks might tend to merge. In 

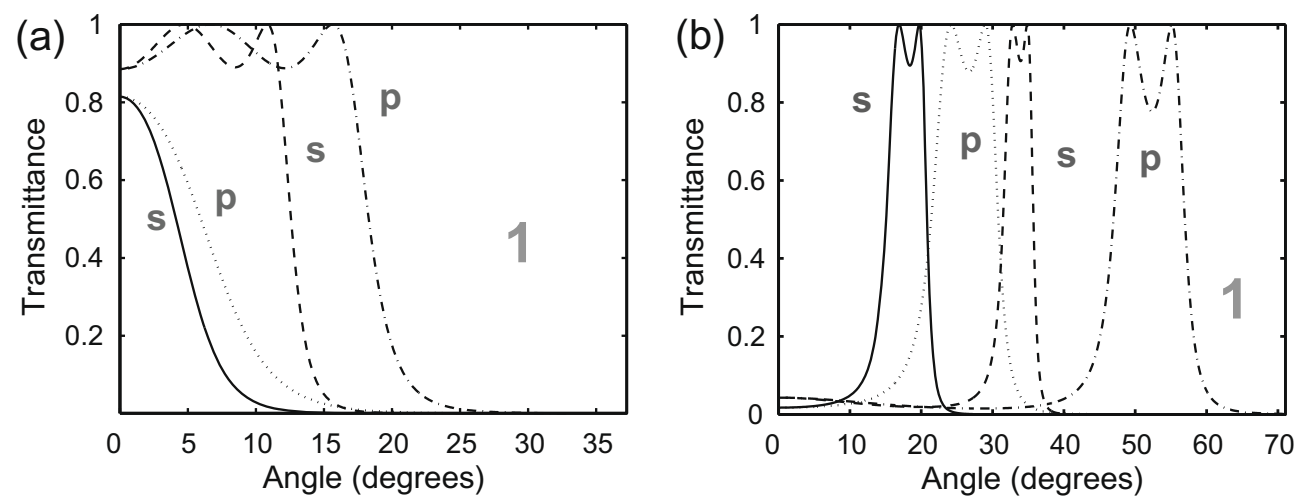

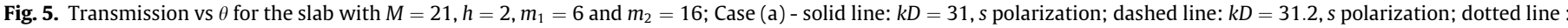

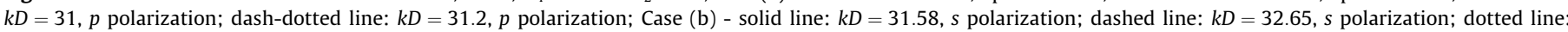
$k D=31.58, p$ polarization; dash-dotted line: $k D=32.65, p$ polarization.
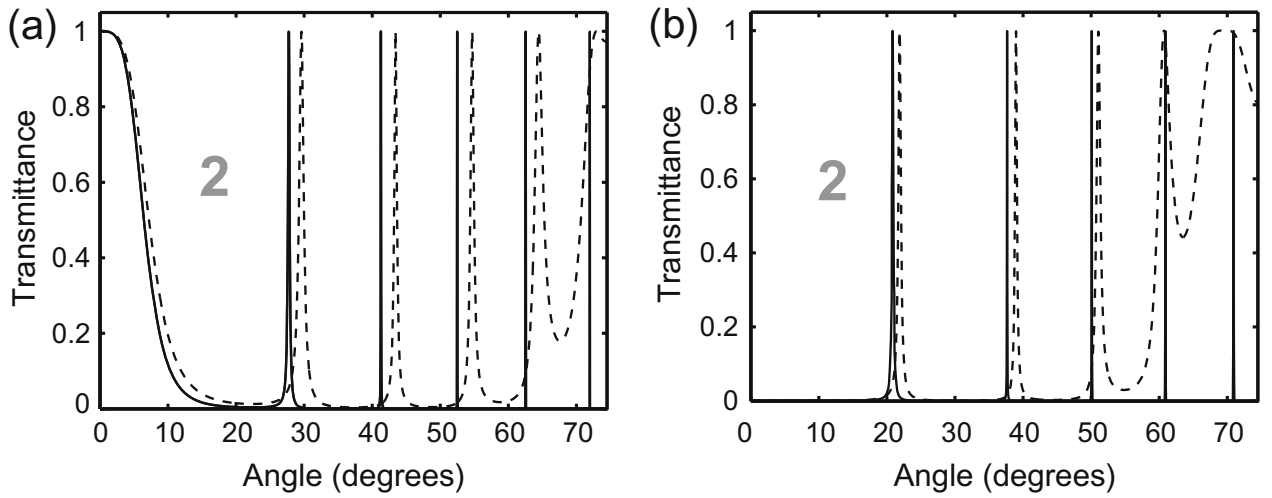

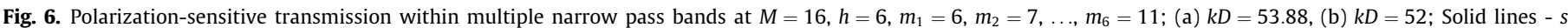
polarization, dashed lines - $p$ polarization.

Fig. $6 \mathrm{~b}$, the $k D$ value is taken beyond the $\omega$-domain pass bands at $\theta=0$, in order to prevent the appearence of a wide $\theta$-domain maximum. Hence, the $\theta$-dependence of $\mathscr{T}$ can also be obtained, which is not typical for Fabry-Pérot etalon. In contrast to Figs. 4 and 5, a multiband $\theta$-domain operation can be realized for this type of the filters at fixed frequency and polarization.

Transmission for regular and defect-mode pass bands of PCs is often interpreted by assigning the equivalent parameters of Fabry-Pérot etalon $[14,23]$. Such interpretations allow one to better understand the basic physics, to bridge a gap between different theories, and are quite natural for periodic lattices with and without defects. Let us attempt to fit the data for $s$ polarization, assuming that the reflections from front- and back-side semitransparent thin planar mirrors are the same. In this case,

$\mathscr{T}=(1-\widehat{R})^{2} /\left[(1-\widehat{R})^{2}+4 \widehat{R} \sin ^{2}\left(n_{m} k b \cos \theta\right)\right]$,

where $\widehat{R}$ is reflection coefficient of a mirror, and $b$ and $n_{m}$ are the distance and index of refraction of the medium between the mirrors, respectively. Generally, there is no unique way to set equivalent parameters. We use here two alternative approaches. In line with the first of them, which is consistent with the interpretation used in [23], the equivalent distance $b_{e q}$ can be introduced regardless of $\widehat{R}$ within a range of $\theta$-variation, which contains $I$ isolated peaks, provided that

$k b_{e q}=\pi\left(\cos \theta_{i}-\cos \theta_{i+1}\right)^{-1}$

does not depend on $i, i=1,2, \ldots, I-1$, and hence on $\theta$. Otherwise, $k b_{e q}$ depends on $\theta$. This fitting approach corresponds to the assump- tion that the medium between mirrors is air, i.e., the equivalent index of refraction $n_{e q}=n_{m}=1$. The obtained values of $k b_{e q}$ are shown in Table 1 for Figs. 6a and 6b. One can see that they vary with $\theta$, but the range of variation is relatively narrow. In terms of transmission, the slab considered mimicks a resonator with planar mirrors, air between them, and equivalent distance between the mirrors that is smaller than the total thickness. On the other hand, $k \Delta=18.97$ in Fig. $6 a$ and 18.3 in Fig. 6b, where $\Delta=h a$, i.e., the width of defect bounded by the equivalent volumetric mirrors, every being $5 a$ thick, is smaller than $b_{e q}$ for all $\theta$.

Alternatively, one can extend the approach from Ref. [14, Chapter 4.4] to the $\theta$-domain, assuming that a homogeneous lossless filling medium may show $n_{e q} \neq 1$. Then, keeping $k b=$ const and

Table 1

Equivalent parameters of Fabry-Pérot etalon, which are obtained from the locations of $\mathscr{T}$-maxima in Fig. 6 in case of $s$ polarization.

\begin{tabular}{lll}
\hline$i, i+1$ & $k b_{e q}$ & $n_{e q}, b=M a$ \\
\hline Fig. $6 a$ & & \\
1,2 & 27.25 & 0.575 \\
2,3 & 23.64 & 0.499 \\
3,4 & 22.0 & 0.464 \\
4,5 & 21.26 & 0.448 \\
5,6 & 20.72 & 0.437 \\
Fig. $6 b$ & & \\
1,2 & 23.75 & 0.519 \\
2,3 & 20.89 & 0.456 \\
3,4 & 20.31 & 0.444 \\
4,5 & 19.64 & 0.429 \\
\hline
\end{tabular}




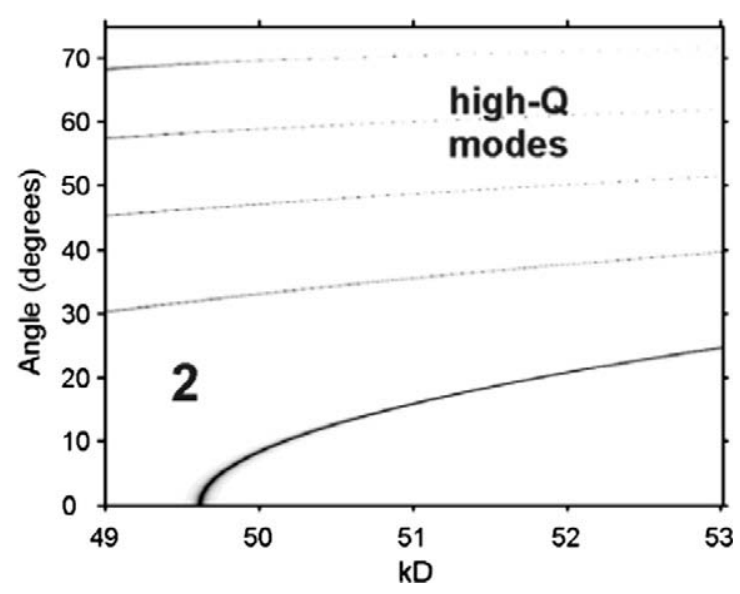

Fig. 7. Transmission vs $k D$ and $\theta$ for the slab with $M=16, h=6, m_{1}=6, m_{2}=7, \ldots$. $m_{6}=11, s$ polarization; the most dark tone in the middle of every line (trace) corresponds to $\mathscr{T}_{s}=1$.

assuming that the identical planar mirrors are located at $x=d_{0}$ and $x=D-d_{0}$, i.e., $b=M a$, we calculate $n_{e q}$ as follows:

$n_{e q}=\pi\left[k b\left(\cos \theta_{i}-\cos \theta_{i+1}\right)\right]^{-1}$.

The obtained values of $n_{e q}$ are presented in Table 1. One can see that $n_{e q}<\varepsilon_{\min }^{1 / 2}$, i.e., the considered graded-index slab mimicks a Fabry-Pérot resonator, which is filled with an ultralow-index material (ULIM), whose $n_{e q}$ depends on $\theta$. The values of $n_{e q}$ substantially differ from the values taken by $n$ within the slab, being connected with the specific dispersion of the used defect modes. Hence, one-dimensional lattices with defects can be used for obtaining Fabry-Pérot resonators with unusual properties. The possibility of mimicking the structures with $n_{e q}<1$ can be used, for example, for avoiding the mode overcrowding and obtaining less dense located peaks of $\mathscr{T}$, especially at large $\theta$. Besides, this example gives an idea of alternative performance of a finite-thickness, purely dielectric optical ULIM, which should be much less sensitive to the restrictions originated from the losses than the existing metallic-wire performances [27]. For the both fitting approaches used, the results only depend on the location of the maxima of $\mathscr{T}$ with respect to each other and do not depend on $\widehat{R}$. It is noteworthy that the presented interpretations are in agreement with the theory of Fabry-Pérot multilayer filters [13].

Note that the number of the peaks in $\theta$-domain, e.g., in Fig. 6 , can be substantially larger than that in $\omega$-domain within the corresponding stop band at a fixed $\theta$, e.g., in Fig. 2 . To explain the causes leading to this situation, we plot $\mathscr{T}$ on the $(k D, \theta)$-plane, see Fig. 7. For the high- $Q$ modes, the traces of the lines corresponding to $\mathscr{T}=1$, which are narrowing at increasing $\theta$, are only seen because the used discretization is not too fine here, being limited by CPU time restrictions. Nevertheless, if one would calculate $\mathscr{T}$ vs $k D$ for a larger number of $\theta$-values than in Fig. 2a, all modes with $\mathscr{T}=1$ can be found from the $\omega$-domain transmission results. However, as seen in Fig. 7, the pass bands correspond to narrow ranges of $\theta$-variation, which are unknown a priori, so that the $\theta$-domain consideration is important, even if the main interest is related to $\omega$-domain. This remains true with respect to the first $\omega$-domain stop band in Fig. 2a. It is worth noting that $d \tilde{\omega} / d k_{d}>0$ for all modes in Fig. 7, and that the $Q$-values up to $3 \times 10^{3}$ can be achieved at a relatively large $\theta$. Besides, note that the $\theta$-domain mini stop bands can appear within a limited range of $k D$-variation. For example, $\mathscr{T} \approx 0$ at $24.5^{\circ}<\theta<30^{\circ}$ and $39.5^{\circ}<\theta<45^{\circ}$, while $k D$ is varied from 49 to 53 .

For a single-band operation, defect modes arising in $\omega$-domain within the first stop band, and hence the slabs with a smaller thickness can be used. $\mathscr{T}$ vs $\theta$ is shown in such a case in Fig. 8a. For the peaks arising at $23.5<k D<24.51, W_{\theta}<0.6^{\circ}$ for $p$ polarization and $W_{\theta}<0.17^{\circ}$ for $s$ polarization. These values are comparable with those required for the angularly tolerant resonant-grating spatial filters [4]. In this case, the structure is about four wavelengths thick. Note that for the two of four $k D$-values used in Fig. $8, \mathscr{T}_{s} \approx 0$ within the whole $\theta$-range considered. Finally, Fig. 8b demonstrates the sensitivity of location of the $\mathscr{T}$-peaks for the second-stop-band defect modes of different polarizations to a slight variation of $k D$.

\section{Conclusions}

To summarize, we have demonstrated a route to the realization of multiband narrow- and wide-bandpass spatial and spatial-frequency filters by using multiple defect modes, which can appear in one-dimensional lattices with the properly introduced defects. The $Q$-values for these modes and locations of the corresponding transmission peaks with respect to each other can be varied in a wide range, leading to various angle-dependent transmission features. In case of the coupled narrow defects (coupled defect modes), several frequencies are involved in the obtaining of a multiband wide-bandpass filter in angle and angle-frequency domains, if only one polarization is used. Both polarizations can be used simultaneously in a similar fashion, so that each frequency value corresponds to an unique range of the angle variation. At fixed frequency, two non-overlapping angle-domain pass bands can be obtained, every corresponding to different polarization. For the
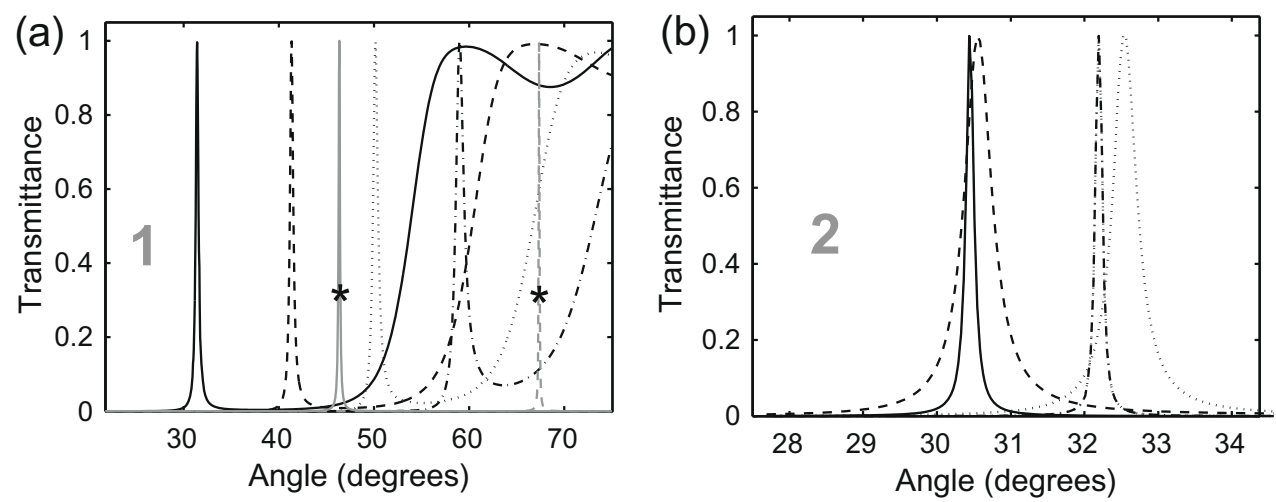

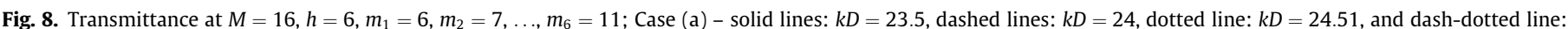

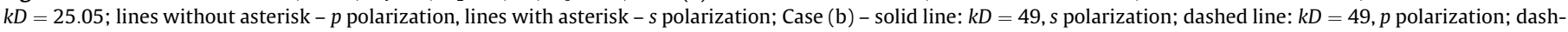
dotted line: $k D=49.61$, $s$ polarization; dotted line: $k D=49.61, p$ polarization. 
permittivity profile containing a single wide defect, multiband narrow-bandpass filtering can be achieved, while every pass band corresponds to one of the multiple defect modes arising at the same frequency. In this case, two approaches have been used for interpretation of the transmission data, which are based on the analogy with Fabry-Pérot etalon. It has been demonstrated that the periodic slabs with defects can mimick Fabry-Pérot resonators, whose equivalent parameters strongly differ from the actual physical parameters of the slab. In particular, transmission associated with an ultralow index of the refraction of the filling medium, which is lower than the minimal value of the index within the profile, can be obtained. This allows one, for example, rarefying the defectmode angle-domain spectrum at large angles. It is expected that the effects observed in the angle domain also appear for some other, e.g., sinusoidal graded-index profiles, and for multilayer piecewise-homogeneous stacks, at least if the basic features of location of the defects and the limits of permittivity variation are kept.

\section{Acknowledgments}

This work was supported in part by the European Union under the projects EU-PHOME and EU-ECONAM, and TUBITAK under the Project Nos. 106E198, 107A004, and 107A012. A. S. thanks TUBITAK for the support provided for this work in the framework of the VSF Program. E. O. also acknowledges partial support from the Turkish Academy of Sciences.

\section{References}

[1] D. Schurig, D.R. Smith, Appl. Phys. Lett. 82 (2003) 2215.

[2] I. Moreno, J.J. Araiza, M. Avedano-Alejo, Opt. Lett. 30 (2005) 914.

[3] R. Rabady, I. Avrutsky, Opt. Lett. 29 (2004) 605.

[4] A. Sentenac, A.-L. Fehrembach, J. Opt. Soc. Am. A 22 (2005) 475.

[5] L. Dettwiller, P. Chavel, J. Opt. Soc. Am. A 1 (1984) 18.

[6] O.F. Siddiqui, G. Eleftheriades, J. Appl. Phys. 99 (2006) 083102.

[7] A.E. Serebryannikov, T. Magath, J. Opt. Soc. Am. B 25 (2008) 286.

[8] A.E. Serebryannikov, A.Y. Petrov, E. Ozbay, Appl. Phys. Lett. 94 (2009) 181101.

[9] B.G. Bovard, Appl. Opt. 32 (1993) 5427

[10] W.H. Southwell, Appl. Opt. 28 (1989) 5091

[11] R.L. Hall, Gradient index bandpass filters, in: Optical Interference Coatings, 1992 OSA Technical Digest Series, vol. 15, Optical Society of America, Washington, DC, 1992, pp. 116-118.

[12] B.G. Bovard, Proc. SPIE 1207 (1990) 218

[13] H.A. McLeod, Thin-Film Optical Filters, IoP Publ., Bristol Philadelphia, 1985.

[14] K. Sakoda, Optical Properties of Photonic Crystals, Springer, Berlin, Heidelberg, New York, 2005

[15] H. Taniyama, J. Appl. Phys. 91 (2002) 3511

[16] M.V. Erementchouk, L.I. Deych, A.A. Lisyansky, Phys. Rev. B 71 (2005) 235335.

[17] S. Lan, S. Nishikawa, Y. Sugimoto, et al., Phys. Rev. B 65 (2002) 165208.

[18] A.C. van Popta, M.M. Hawkeye, J.C. Sit, M.J. Brett, Opt. Lett. 29 (2004) 2545

[19] F. Fedele, J. Yang, Z. Chen, Opt. Lett. 30 (2005) 1506

[20] J. Xia, A.K. Jordan, J.A. Kong, J. Opt. Soc. Am. A 11 (1994) 1081.

[21] T.J. Cui, C.H. Liang, IEEE Trans. Antennas Propag. 42 (1994) 621

[22] T. Magath, A.E. Serebryannikov, J. Opt. Soc. Am. A 22 (2005) 2405.

[23] J.H. Wu, L.K. Ang, A.Q. Liu, H.G. Teo, C. Lu, J. Opt. Soc. Am. B 22 (2005) 1770.

[24] M. Bayindir, B. Temelkuran, E. Ozbay, Phys. Rev. Lett. 84 (2000) 2140.

[25] Y. Othera, T. Sato, T. Kawashima, T. Tamamura, S. Kawakami, Electron. Lett. 35 (1999) 1271.

[26] S. Foteinopoulou, A. Rosenberg, M.M. Sigalas, C.M. Soukoulis, J. Appl. Phys. 89 (2001) 824

[27] B.T. Schwartz, R. Piestun, J. Opt. Soc. Am. B 20 (2003) 2448. 